\title{
Raten Sie Schwangeren von Cannabis ab!
}

\author{
In vielen US-Bundesstaaten und bald auch in Deutschland wird Cannabis als Medikation etwa gegen \\ Übelkeit eingesetzt. Experten fordern nun, dass Schwangere vorerst darauf verzichten sollen.
}

\begin{abstract}
_ Vor dem Hintergrund der fortschreitenden Legalisierung von Medizinalhanf kursieren im Internet Empfehlungen, den pflanzlichen Wirkstoff auch gegen Schwangerschaftsübelkeit einzusetzen. Gleichzeitig ist Cannabis ist in den USA die am häufigsten konsumierte Droge in der Schwangerschaft. US-Forscher des National Institute on Drug Abuse haben nun die Datenlage zu den damit einhergehenden Risiken diskutiert.

Die Einflüsse von Cannabis auf die Entwicklung im Mutterleib sind unzureichend untersucht. Allerdings gibt es Hinweise auf ein erhöhtes Risiko für Anämie, niedriges Geburtsgewicht und die Notwendigkeit einer intensivmedizinischen Überwachung der Neugeborenen. Zudem wurde eine Beeinträchtigung höherer Exekutivfunktionen wie
\end{abstract}

Impulskontrolle, visuellem Gedächtnis und Aufmerksamkeit gefunden.

In Mausversuchen mit den seit wenigen Jahren verfügbaren synthetischen Cannabinoiden, die noch stärker mit den Cannabinoidrezeptoren interagieren, konnten teratogene Effekte gezeigt werden. Je höher die Dosis war, desto mehr Mäuse kamen fehlgebildet zur Welt. Bei Menschen gibt es dagegen noch keine ähnlichen Hinweise.

Die Autoren fordern weitere Untersuchungen, insbesondere mit hochdosierten THC-Präparationen und synthetischen Cannabinoiden. Einstweilen sollten Ärzte ihren schwangeren Patientinnen aktiv vom Cannabiskonsum abraten. Selbst zur Behandlung von Hyperemesis gravidarum könne Medizinalhanf derzeit nicht empfohlen werden.
- Volkow ND, Compton WM, Wargo EM. The risks of marijuana use during pregnancy. JAMA. 2017;317:129-30

\section{KOMMENTAR}

Neue wissenschaftliche Umfragen unter Frauen zeigen, dass diese auch in der Schwangerschaft Cannabis konsumieren. $10 \%$ von ihnen gaben an, dass sie auch mehr konsumieren würden, wenn es legal wäre [Mark K et al. J Addict Med 2017, online 1. März]. Bis es belastbare Daten gibt, sollte Frauen mit Kinderwunsch und Schwangeren dringend vom Konsum von Cannabis- und insbesondere Cannabinoidprodukten abgeraten werden. Gegen Schwangerschaftsübelkeit gibt es weit besser untersuchte Mittel, z. B. Dimenhydrinat oder Mirtazapin [Abramowitz A et al. Arch Womens Ment Health 2017, online 9. Januar].

Dr. med. S. Kittel-Schneider

\section{Eine Riesennase mit krustigen Zacken}
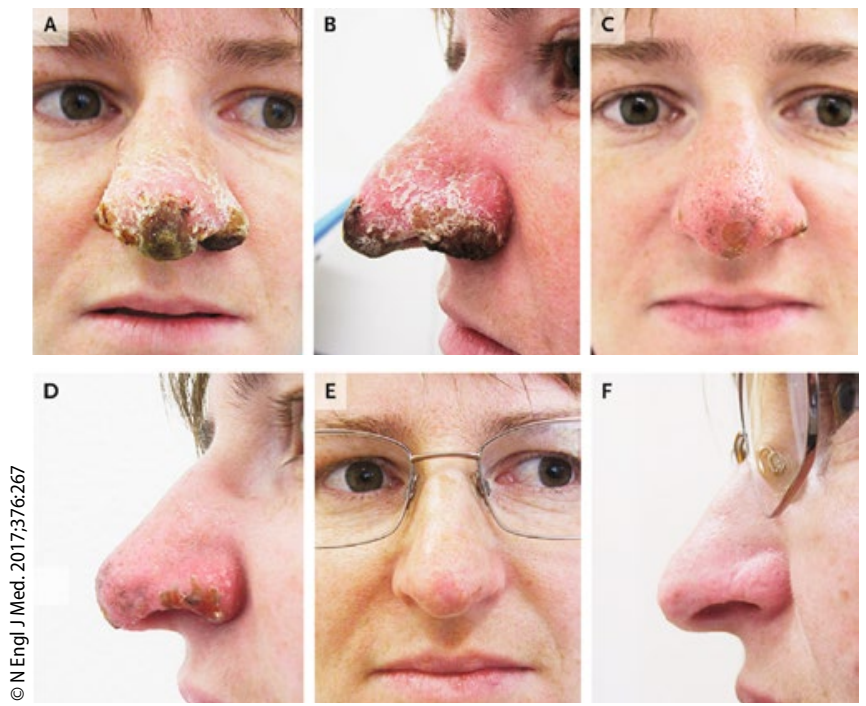

A, B: Geschwollene, ulzerierte Nase. C, D: Erfolg der ersten Antibiotikatherapie. E, F: Vollständige Abheilung nach der zweiten Antibiotikatherapie.
Eine 37-jährige Frau stellte sich nun wegen einer seit sechs Wochen bestehenden Schwellung der Nase mit Ulzerationen vor (Abb. A, B). In einer Hautbiopsie aus dem Ulkus konnte Serratia liquefaciens kultiviert werden. Eine dreiwöchige Behandlung mit Ciprofloxacin führte zu einer gewissen Besserung (Abb. C, D). Fünf Wochen später war in der Kultur der Biopsien Mycobacterium marinum gewachsen, dem Erreger des Aquarium-Granuloms. In den Biopsien hatte man auch verkäsende Granulome gefunden. Man behandelte sieben Monate lang mit Clarithromycin und Ethambutol. Sechs Monate danach waren die Läsionen abgeheilt (Abb. E, F).

Beide hier kultivierten Mikroorganismen können im Wasser gefunden werden. Wie es bei der Patientin zu der Infektion im Bereich der Nase kam, blieb unklar. Zwei Monate vor der Erstvorstellung hatte sie ein steroidhaltiges Nasenspray verwendet und sich einer Verkleinerung der unteren Nasenmuschel mittels Kauterisierung unterzogen. Möglicherweise hing die Infektion auch mit ihren regelmäßigen Besuchen in einem Thermalbad zusammen.

Prof. Dr. med. H. S. FüeßI

- Schlapbach C, SendiP (christoph.schlapbach@insel.ch). Serrated marine nose. N Engl J Med. 2017;376:267 